\section{Der Obstanbau auf den Ländereien der Gutsherrschaft REINHARDTSGRIMMA}

Susan Mauersberger

A s auf dem Rittergut Reinhardtsrimma der systematische Obstbau 1800 unter der Familie von Bülow begann ${ }^{1}$, konnte Sachsen bereits als das drittgrößte geschlossene Obstanbaugebiet Deutschlands angesehen werden. Erfolgte der richtungsweisende Anbau davor hauptsächlich auf »Königsgütern«, verbreitete sich im 19. Jahrhundert der Obstanbau und begann die hiesige Landschaft nachhaltig zu prägen. ${ }^{2}$ Aufgrund der hohen Priorität des Obstbaus stellt sich die Frage, wie sich dies beispielsweise auf das Land und die Bevölkerung niederschlug - wirtschaftlich wie auch kulturell. Wie wurden Erkenntnisse ausgetauscht? Und weshalb erfreute sich die Beschäftigung mit den Früchten ausgerech net beim Adel so großer Beliebtheit? Ja, wie sah dies speziell in Reinhardtsgrimma aus?

\section{Die Bedeutung und Entwicklung der Pomologie}

Die Pomologie beinhaltet die Lehre von den Obstarten und -sorten, sowie deren Bestimmung und Einteilung. Jedoch ist die Pomologie keineswegs als trivial anzusehen, wird sie doch auch als `soziologisch ‘ beschrieben. Welche Obstsorten in einem Garten kultiviert wurden, hing vor allem vom gesellschaftlichen Stand ab. So fand man in einem Bauerngarten andere Kulturen vor wie in einem Bürger-, Pfarr-, Kloster- oder gar einem Schlossgarten. Die Einführung neuer Kulturen fand grundsätzlich durch die führende Gesellschaftsschicht statt. Das Bürgertum übernahm die Neuerungen bereitwillig; die einfachen Leute hingegen sträubten sich zuweilen, da man sich gern mit >Altbewähr- tem $<$ bei möglichst wenig Aufwand begnügte und erst unter Druck nachgab. Die andesherren und der Adel andrerseits glänzten mit den neuesten Sorten, deren Beschaffung und Anbau zumeist mit einem horen Aufwand verbunden war. Aus diesem Grund sollte bei der Wiederbepflanzung eines historischen Gartens der gesellschaftliche Stand des früheren Besitzers berücksichtigt werden. So wäre eine Lokalsorte in einem Schlossgarten einfach unpassend! ${ }^{3}$

Auf dem Rittergut Reinhardtsgrimma wählte man einen Mittelweg, wie Aufzeichnungen zu den Beständen verraten. ${ }^{4}$ So wuren nicht nur besondere und wohlschmeckende Sorten angebaut, sondern auch solche, welche sich als Wirtschaftsobst und somit zur Verarbeitung eigneten.

Verordnungen die Obstkultur betreffend, lassen sich bereits unter Karl dem Großen (747/48-814) finden, da die Früchte einen Beitrag zum Lebensunterhalt leisten sollten. Träger der Kenntnisse über Zucht und Verbreitung waren in der Regel die Klöster. So hat sich seit dem 17. Jahrhundert eine pomologische Terminologie entwickelt, die bis zum heutigen Tag Gültigkeit hat. In der Fachsprache unterschied man zu jener Zeit vier Baumformen »formae arborum pomiferarum «": 1. Hochstamm, 2. Wandspalier, 3 Freistehendes Spalier, 4. Zwergbaum. Auch gab es die Unterteilung in drei Höhenklassen: Hochstamm, Halbstamm und Nieder stamm. Veredlungsversuche an Niederstämmen führten $\mathrm{zu}$ raschen Erfolgen und $\mathrm{zu}$ besseren Erträgen als bei Hochstämmen, dies begünstigte auch die Auslese der Sorn. Das Formobst wie man es im ausgehen- den 17. Jahrhundert für die Kultur in baumveredlung im Gartenbau diente und Deutschland entdeckte, nahm seinen Ur- im weiteren Sinn ein Lehrbuch über versprung auf dem europäischen Kontinent in schiedene Arbeiten des Obst- und Wein-

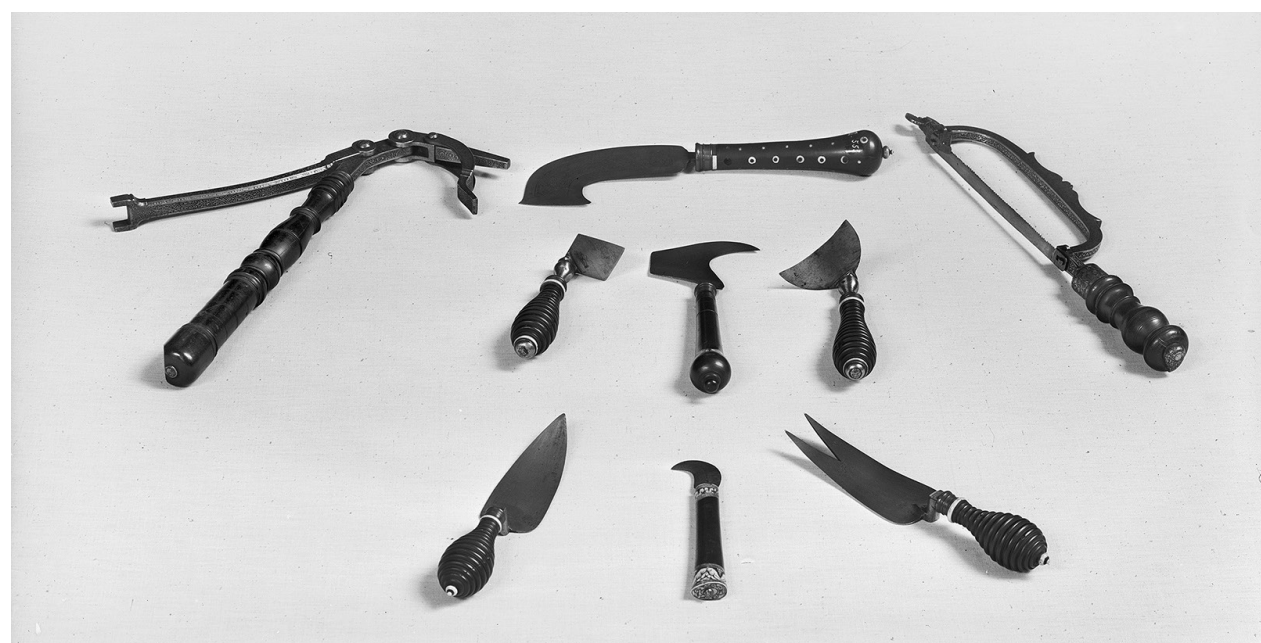

1 | Gartenwerkzeuge des Kurfürsten August von Sachsen, 16. Jh., SKD - Bestand der Rüstkammer, Fotografie Walter Möbius, 1938 (Deutsche Fotothek, df_hauptkatalog_0058995). Frankreich, ein Vorbild, das sich auf ganz Europa auswirken sollte. ${ }^{6}$

Im wirtschaftlich stabilen Sachsen erwiesen sich im 16. Jahrhundert Kurfürst August 1526-1586) und dessen Frau, Kurfürstin d Anna (1532-1585), als besondere Förderer des Obstbaus. Der Kurfürst veredelte eigenhändig und gab später auch Gehölze an ihm nahestehende Fürsten weiter. So erließ August 1560 den Codex Augusteus? . Dieser wies Bauern dazu an »uf ihre Güter wilde ObstStämme $\aleph^{8}$ zu pflanzen. Auch wurde die Einführung neuer Obstsorten und der Austausch von Reisern veranlasst sowie Tauschhandel mit Sämereien wie Obstsamen gegen Getreidesaat betrieben. Kurfürst August ließ drei große Baumschulen bzw. Obstgärten im Dresdner Umfeld, bei Torgau, Stolpen und Lochau - dem späteren Annaburg - anlegen. Hinzu kam die Musterwirtschaft des Kurfürsten im Vorwerk Ostra. ${ }^{9}$ Unterdessen betrieb Anna regen schriftlichen Austausch mit Beamten und Herrschern über den Obst- und Gemüseanbau. Der Kurfürst publizierte zudem ein sogenanntes Pelzbuch

eine Schrift, die der Vermittlung der Obst-

baus darstellte. Der Inhalt befasste sich demnach mit »dem praktischen Gartenbau, aber nicht mit der Sortenkunde « ${ }^{10}$ (Abb. 1)

Im ersten Drittel des 17. Jahrhunderts brach er Dreißigjährige Krieg (1618-1648) über Deutschland herein. Doch was bedeutete dieses Ereignis voller Entbehrung und Zerstorung für den sächsischen Obstbau? Während zu Anfang des Krieges noch eine Neuauflage des Apfelbüchleins ${ }^{11}$ von Kurfürs sen im Lauf der Jahrzehnte mehr und mehr zum Erliegen. Nach Kriegsende unternahm man erste Wiederbelebungsmaßnahmen, die sich u.a. auf das sächsische EhestandsBaumgesetz stützten, welches »Vater August « 1577 erlassen hatte. Dieses beinhaltete Pflanzen und Pflegen zweier hochstämmiger Obstbäume auf der Gemeindefläche auferlegt wurde. Diese Gesetzmäßigkeit erhielten nachfolgenden Landesherren aufrecht und bauten sie weiter aus. ${ }^{12}$ Bis weit nach Kriegsende galt der Hochstamm als einzige Kulturform in Deutschland. ${ }^{13}$ August erschien, kam der Obstbau in Sach- 


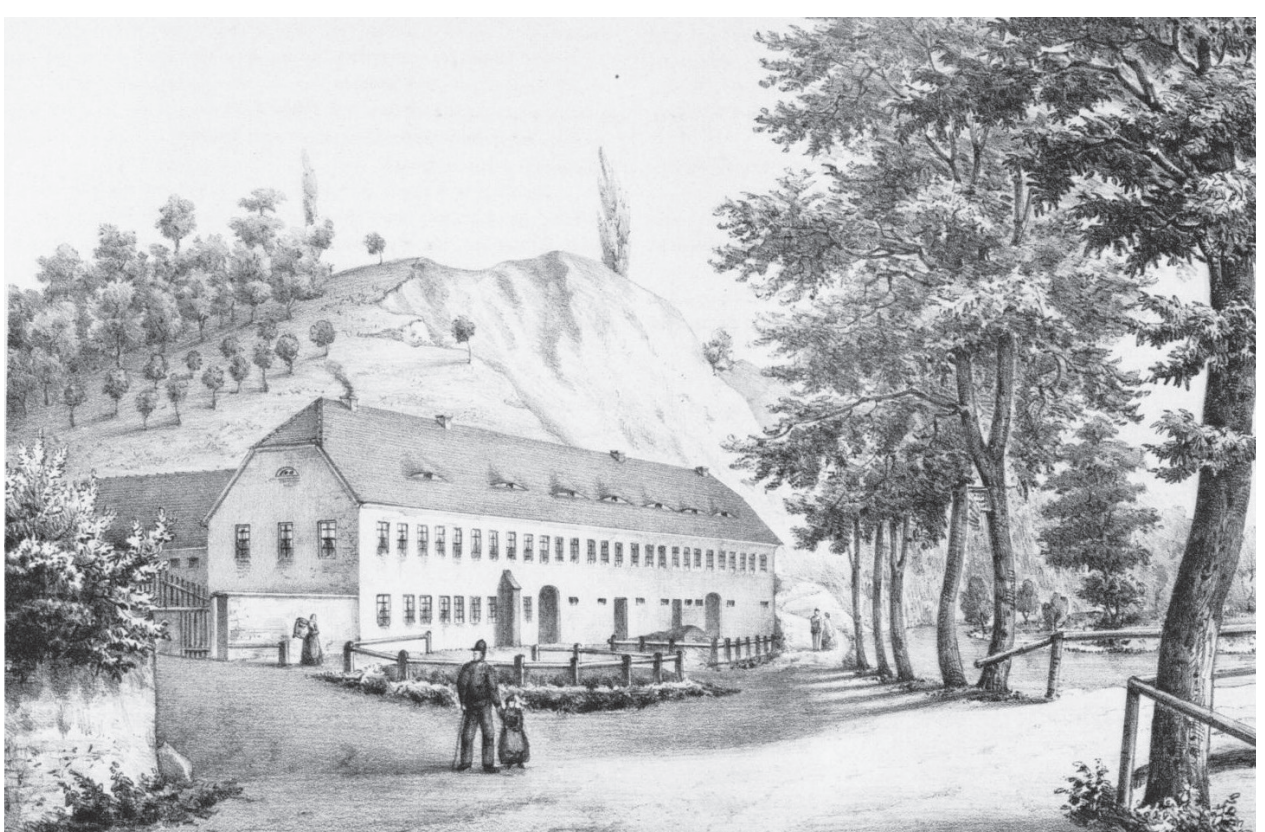

2 | Das Rittergut Potschappel bei Dresden im 19. Jahrhundert. Der Hang im Hintergrund ist mit einer Obstplantage bestückt. Lithografie (aus Poenicke, Gustav Adolf: Album der Schlösser und Rittergüter im Königreiche Sachsen II, Meissner Kreis, Band 31, Leipzig 1856, Abb. zu Beitrag Potschappel ab S. 211).

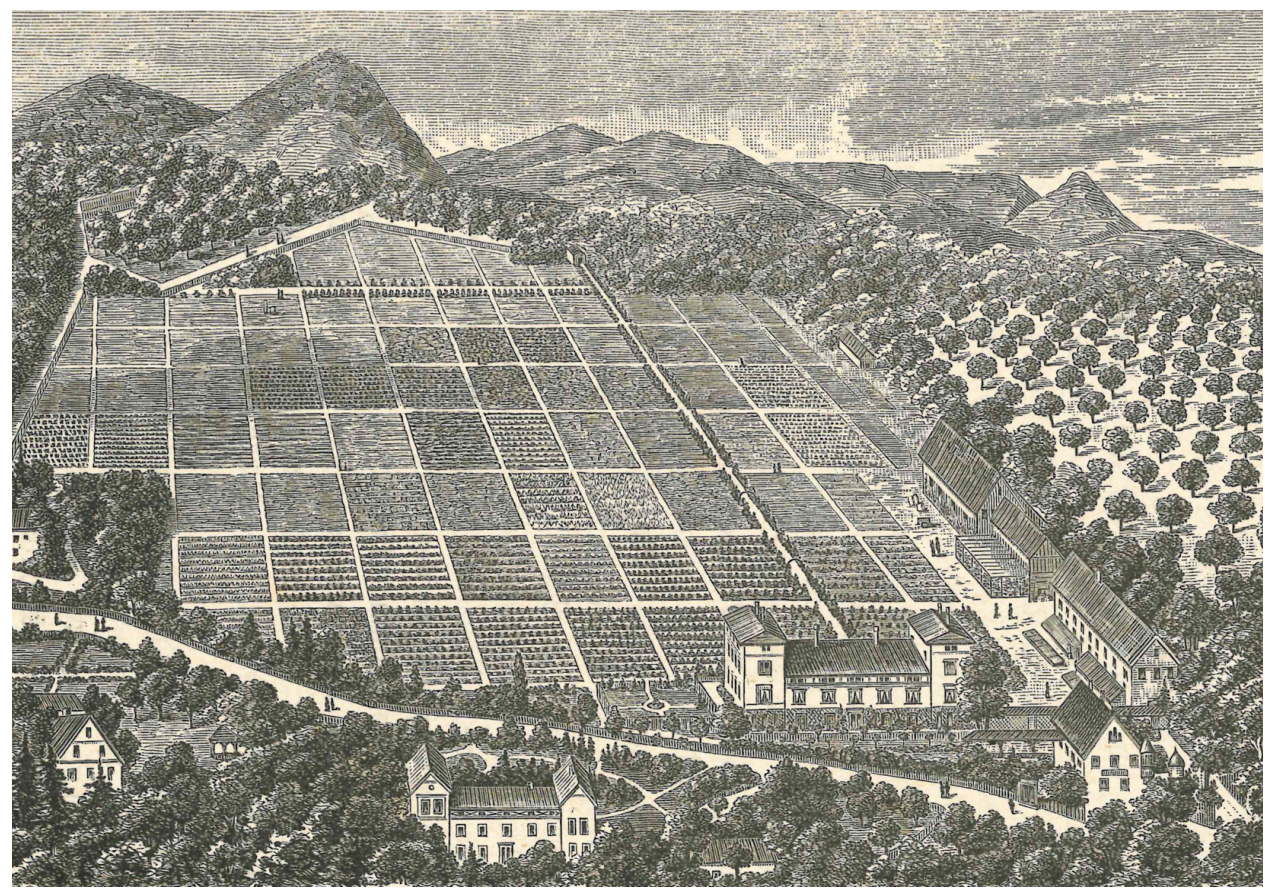

$3 \mid$ Der erste Pomologische Garten in Reutlingen um 1885, gegründet durch Eduard Lucas. Im Vordergrund das Pomologische Institut mit Baumschule, Obstmustergarten, Arboretum und Beerenobstgarten (aus Lucas, F. Pomologisches Monatsheft, Band 31, Stuttgart 1885, S. 119).
Bis zum Ende des 17. Jahrhunderts diente sächsische "Nationalfrucht« an. So galt der der Obstbau vor allem der Selbstversorgung >Borsdorfer`bis ins 17. Jahrhundert, nach Erund weniger dem Marktanbau, wenn auch kenntnissen des bekannten Pomologen überschüssige Ware verkauft wurde. ${ }^{14} \operatorname{Im} 18$. Eduard Lucas (1816-1882), als beste deutsche Jahrhundert bemühten sich die sächsischen Apfelsorte. ${ }^{20}$

Herrscher weiter um die Förderung des

Obstbaus. So predigten Pfarrer unter Fried- 1855 erfolgte die Gründung der von Eduard rich August I. (1670-1733), bekannt als »Au- Lucas herausgegebenen »Monatsschrift für gust der Starke«, in den Gottesdiensten von Pomologie und praktischen Obstbau « ${ }^{21}$ soder Kanzel unermüdlich, um die Pflanzung wie 1860 die Gründung seines Pomologivon fruchtbaren Bäumen auf Anwesen und schen Institutes in Reutlingen, das bis 1921 Grundstücken zu fördern, erwiesen sie sich bestand und den ersten pomologischen Gardoch nicht selten selbst als leidenschaftliche ten überhaupt aufwies (Abb. 3). 1857 erweiObstkundler. Sie sahen in der Förderung des terte man zudem das Normsortiment von Obstbaus eine wichtige Aufgabe und trugen Äpfeln und Birnen und trieb die Herausgabe viel zu dessen Verbreitung unter den Bür- eines Obstbauhandbuches voran. Das Begern und Bauern bei.

streben der Obstkunde war die vollständige Erfassung und Systematisierung des SortenDer Siebenjährige Krieg (1756-1763) in der bestandes, was allerdings aufgrund der stänMitte des 18. Jahrhunderts führte noch ein- dig wachsenden Vielfalt an Sorten eher mämal zu herben Verlusten des Baumbestandes Big funktionierte. Mit dem Bemühen, sich in Sachsen. ${ }^{15}$ Dieser erholte sich jedoch nicht nur praktisch mit der Materie auseinschnell. 1819 erschien das »Verzeichnis der anderzusetzen, sondern auch wissenschaftObstsorten in der systematischen Obst- liche Erkenntnisse zu verbreiten und über baumschule im königl. Sächs. großen Gar- Neuerungen zu informieren, erreichte der ten bei Dresden. ${ }^{16}$ Dresdens "Großer Gar- Obstbau seinen Höhepunkt. Denn bei der ten « lag zu Anfang des 19. Jahrhunderts Monatsschrift von Lucas handelte es sich noch jenseits der Stadtmauern. ${ }^{17}$ Auch glie- keineswegs um ein dünnes Magazin, sonderte man um 1830 eine weitere Obstbaum- dern um ein fast vierhundert Seiten umfasschule im Forstgarten der Königlichen sendes starkes »Bändchen«, das auch die LeForsthochschule Tharandt ein. In Städten serschaft einbezog, indem man auf Anfraund Gemeinden begann man kommunalen gen einging sowie deren Anmerkungen und Obstbau zu betreiben, da dieser sich durch neue Erkenntnisse abdruckte, sofern sie indie Verpachtung als eine sichere Einnahme- teressant erschienen. quelle erwies. Der landwirtschaftliche Apfelanbau erfolgte auf Rittergütern und deren Nach einer sächsischen Rekordernte um dazugehörigen Domänen (Abb. 2). Zudem $1850^{22}$ dämpfte sich der obstbauliche Optientwickelten sich die Pomologischen Gärten mismus gegenüber Äpfeln allmählich, da von dezentralen Sortensammlungen zu diese aufgrund fehlender Verwertung zu unwahren Mustergärten, wie beispielsweise in rentablem Viehfutter verkamen. Dies führte Görlitz oder dem sächsischen Leißnig. ${ }^{18}$ Ins- aber auch zur Entwicklung der industriellen piriert durch die Obstgärten der Benedikti- Obstverwertung etwa durch die Freiherr ner und Zisterzienser galten sie dem Land- von Friesensche Gartendirektion in Rötha adel und den Bauern als Vorbild. So wurden bei Leipzig, denn die Getränkeerzeugung Äpfel vor allem in höfischen Gartenanlagen wurde zu diesem Zeitpunkt immer wichtirund um Dörfer und Städte gepflanzt. ${ }^{19}$ Als ger. Friedrich Otto Heinrich Freiherr von älteste bekannte und beliebte Apfelsorte Friesen (1831-1910), dessen Gutswirtschaft Mitteleuropas baute man den $>$ Borsdorfer auf den Obstbau ausgerichtet war, leistete Apfel« sowie die `Teutsche Pomeranze`, einst Bedeutendes: Neben der Schlossgärtnerei 
auf Schloss Rötha gründete er 1871 eine neue Gärtnerei mit Gärten, Plantagen, Wegen

und Feldrändern, die mit Obstgehölzen be- Für eine Etablierung des Obstbaus als Wirtstückt wurden. Er war Verfasser der Werke schaftszweig waren Baumschulen und gute »Die Anpflanzung von Bäumen an den öf- Verkehrsanbindungen Voraussetzung. Da fentlichen Wegen « (1878) sowie des »Practi- sich die großen Baumschulen in Holland schen Führers im Obstbau« (1881). und Frankreich befanden, gründeten Lan-

Neben der verbesserten Obstverwertung desherren und Handelsgärtner im 18. Jahrbot man nun auch Fachausbildungen an, hundert in ihrer jeweiligen Region zahlreiwofür 1875 die erste Gärtnerlehranstalt für che Baumschulen und Handelsgärtnereien Obst- und Gartenbau zur Aus- und Weiter- (Abb. 5) um den Bedarf decken zu können. bildung von Baumwarten, Lehrlingen und Da der Transport größerer Gehölze durch Gehilfen bei Rötha in Sachsen eröffnet schlechte Straßenverhältnisse mühselig war, wurde. ${ }^{23}$ Obst wurde Handelsobjekt und beschränkte man sich auf Reiser, da diese für Grundnahrungsmittel zugleich. So entstand den längeren Transport gut geeignet waein starker Impuls für die Verwendung von ren. ${ }^{27}$ Eine Unterlage zur Pfropfung zog man Obst zu nichtalkoholischen Getränken. Es sich vor Ort selbst. Zu unterteilen waren bildeten sich Foren zum Erfahrungsaus- drei verschiedene Arten an Obst: Das Tafeltausch. Landesobstbauinspektoren, Kreiso- obst, gemeinhin von hoher Qualität und bergärtner und Kreisbaumwärter übernah- ohne weitere Zubereitung zum Verzehr gemen die Beratung in der Praxis. Um 1920 eignet, kam aus dem Obstgarten oder existierten landesweit 111 Obstbauvereine, stammte von den am Haus wachsenden Spasowie acht Lehranstalten, Institute und Ver- lieren. Das Wirtschaftsobst, das zur weiteren suchsanlagen sowie 38 mustergültige Obst- Verarbeitung vorgesehen war und sich anlagen. Verschiedene Obstfachleute hatten durch mindere Qualität nicht als Tafelobst amtliche Stellungen inne. ${ }^{24}$ eignete, kam von den Streuobstwiesen und Obstbaumalleen. Genutzt wurde dieses als Ein Schwerpunkt - vor allem auch der säch- Lager- oder Trockenobst, als Mostobst, sischen Pomologie im 19. Jahrhundert -, lag Kochobst oder Einmachobst sowie als Futdarin, geeignete Sorten für verschiedene terobst für das Vieh. War Wirtschaftsobs Standorte auszuprobieren und zu empfeh- von besonders minderer Qualität, so galt len. Daneben wird aber auch deutlich, dass dieses als Industrieobst und wurde als Roh man vor allem den Obstbau an Wegen und stoff zur Gewinnung von NahrungsmittelStraßen förderte. Die einfache Pflege der zusatzstoffen ${ }^{28}$ wie Fruchtzucker, Pektin Bäume, auf Grund der guten Erreichbarkeit, oder Industriealkohol genutzt ${ }^{29}$. aber auch der Transport des dort angebau-

ten Wirtschaftsobstes mittels der zuneh- Der Obstanbau auf dem Rittergut in mend ausgebauten Straßen schien neue Reinhardtsgrimma bis 1850 wirtschaftliche Chancen zu eröffnen ${ }^{25}$ Diese

Initiativen waren erfolgreich, wie die An- Nachweislich begann der Obstanbau in fang des 20. Jahrhunderts durchgeführte Reinhardtsgrimma bereits unter Johann erste Reichsobstbaumzählung vermittelt. ['Hanß'] Georg von Osterhausen (1577Allein in Sachsen zählte man 7,1 Millionen 1627). Zu diesem Zeitpunkt unterteilte man Obstgehölze, deren Anbau durch staatliche den Ort noch in Ober- und Niederhof und Beihilfen gefördert wurde. 1924 erschien das benannte einen »Bergkgartteun« und einen »Obstsortenverzeichnis für Sachsen « mit »Herrengarteun «.30 Zum Anbau von Obst 144 Sorten. Zusätzlich förderlich waren die und Rüben nutzte man den Schloss- sowie obligatorische Bienenhaltung (Abb. 4) und den Krautgarten auf dem Oberhof ${ }^{31}$, aber der Windschutz um die Obstanlagen. ${ }^{26}$

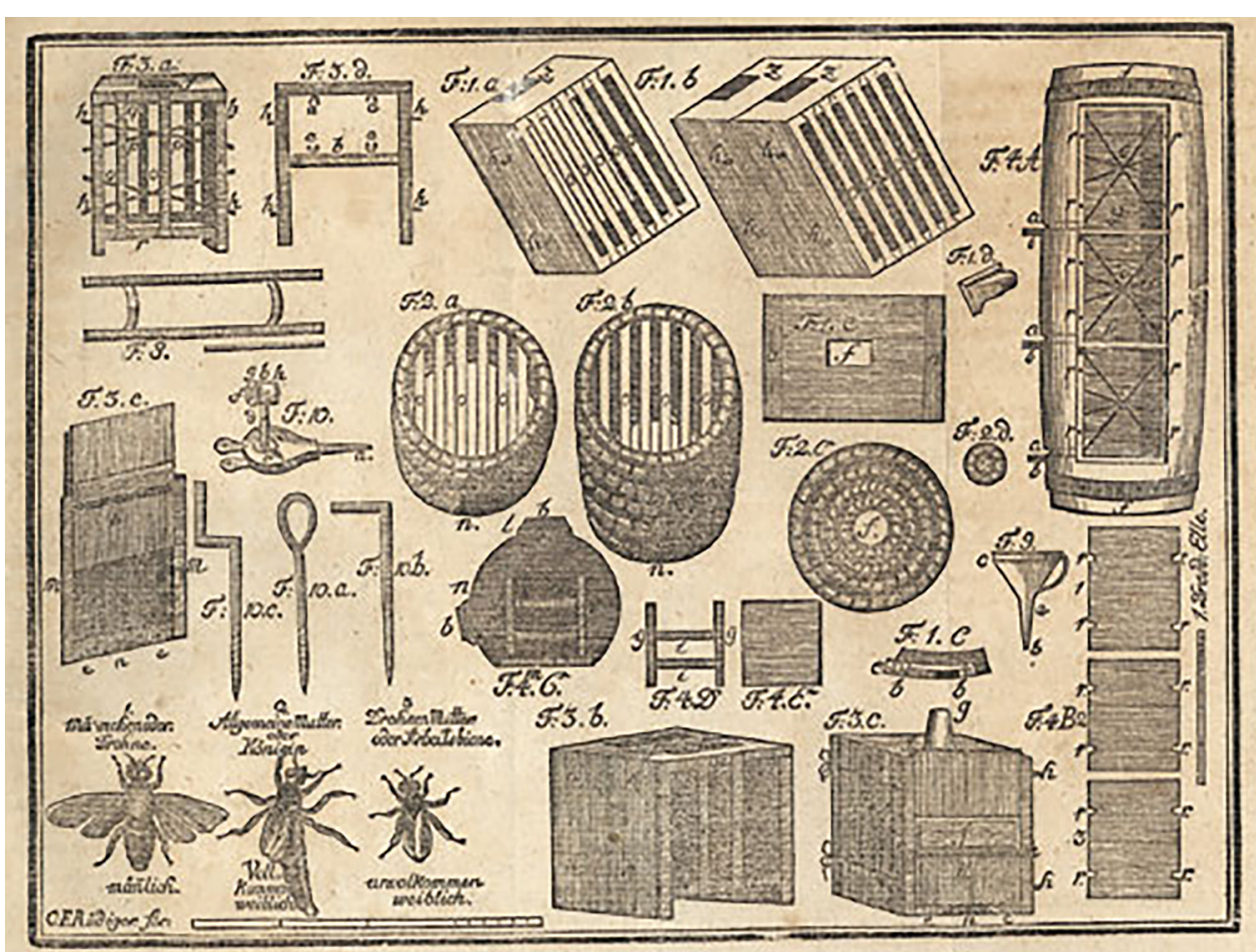

4 | Imkereizubehör um 1820, eine Grundausstattung für Bienenhaltung auf den Obstplantagen im 19. Jahrhundert (aus Riem, Johannes; Werner, Johann Ernst: Der praktische Bienenvater. 4. Auflage, Leipzig 1820, S. 240)

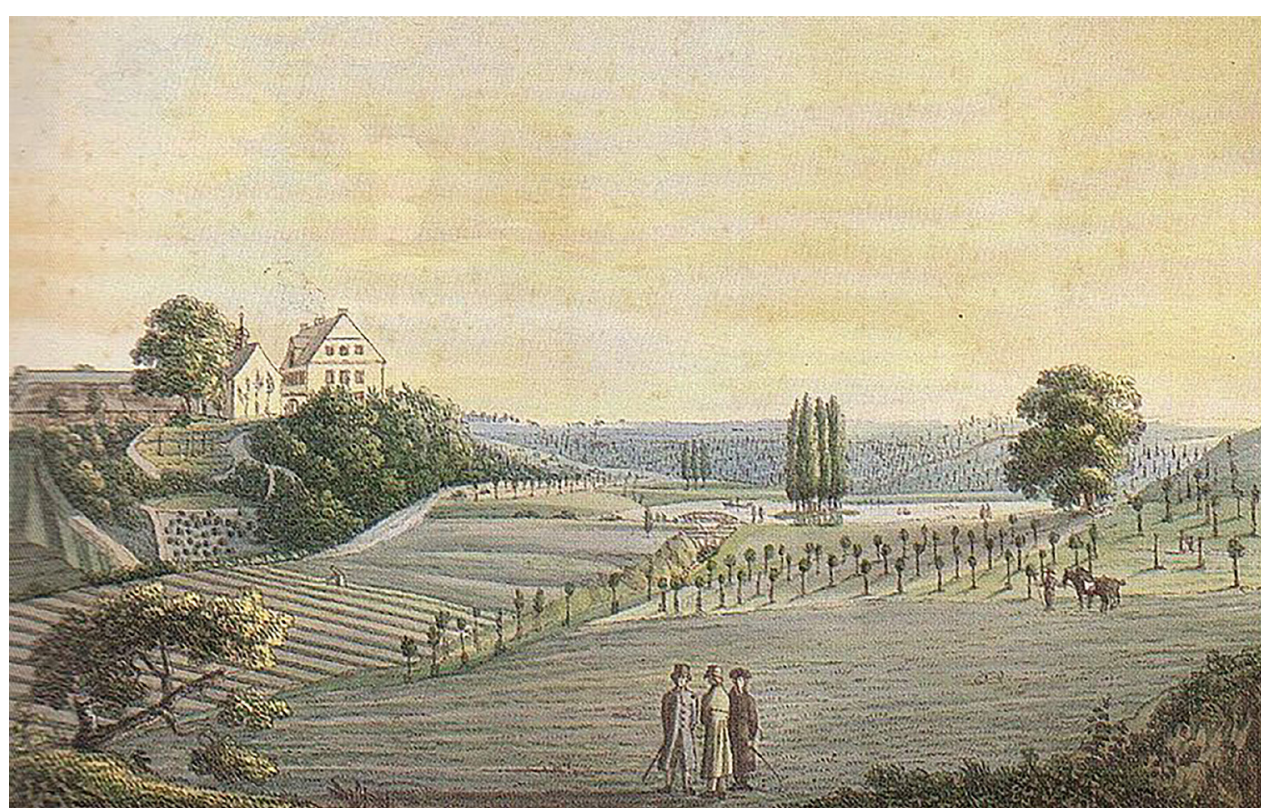

5 Malerische Ansicht der Handelsgärtnerei von Althaldensleben bei Magdeburg um 1820. Sie zählte zu den bedeutendsten Baumschulen Mitte des 19.Jahrhunderts in Deutschland. Farbige Lithografie (https://commons. wikimedia.org/wiki/File:Gl\%C3\%BCsig,_Stich2.JPG, Stand: 04.03.2020). 
oder der Schäferei ${ }^{32}$. Obschon weitere De- den Herrschaften angehalten, kein Obst vertails fehlen, kann man davon ausgehen, dass kommen zu lassen, den richtigen Punkt der - wie spätere Pachtverträge von 1716 und Reife abzuwarten und das Obst nach der 1731 belegen - wertvolles Obst im Schloss- Abnahme unverzüglich zur weiteren Verargarten kultiviert wurde, hingegen man soge- beitung zu übergeben. ${ }^{36}$

nanntes "Wildes Obst« an Wegen und der- Dass die herrschaftliche Küche damals gleichen pflanzte ${ }^{33}$ (Abb. 6). Da der Pfle- Obst schätzte, beweist der von Johann Friedgeaufwand dieser Gehölze aufwendiger war rich Knöbel um 1767 verfasste Grundriss des und es immer wieder zu Diebstahl kam, Kellergewölbes, in dem ein separater Raum wurde der Gärtner prozentual an der Ernte als »Cave pour le fruits et les herbes « geführt beteiligt, wohingegen das andere Obst aus- wird (Abb. 7)..$^{37}$

schließlich der Herrschaft gehörte. Dies

zeigte sich noch einmal deutlich, als 1809 Obschon die Akten aus der ersten Hälfte des zwischen dem neuen Herrschaftsgärtner Jo- 19 . Jahrhunderts nur spärliche Informatiohann Friedrich Reuter und der Gutsbesitze- nen liefern, kann man davon ausgehen, dass rin Friedericke Juliane Christiane Freiin von mit dem Förster Georg Conrad RuschenBülow ein Vertrag ${ }^{34}$ mit folgenden Bedin- busch, der 1802 nach Reinhardtsgrimma gungen ausgehandelt wurde: So war der kam und das Gut 1830 kaufte, den ForstGärtner zuständig für die Kultivierung aller und Obstbäumen ein besonderes Interesse vor Ort befindlichen Obstbäume in- und entgegenkam..$^{38}$ Als Mitglied der Leipziger außerhalb der Gärten. Bei der Baumpflege Ökonomischen Soezietät war er über den standen ihm Gehilfen und Tagelöhner zur Stand der Pomologie gut unterrichtet und Verfügung, wobei er aufgefordert wurde, folgte wohl auch deren Zielen die Guts-, "sowohl des Nutzens als das Beyspieles we- Haus- und Volkswirtschaft zu verbessern. ${ }^{39}$ gen, selbst mit Hand anzulegen ${ }^{35}$. Auch be- Insgesamt befand man sich aber auch dort kam er mit seinem Mitstreiter, dem Gärtner erst in einer Anfangsphase, heißt es doch in Helbig, gemeinschaftlich ein Viertel der einer Schrift von 1830

Pachtsumme für sämtliche verpachteten

Obstgehölze, die sich außerhalb des Gutes

befanden. Zudem waren die Gärtner von

»Wenn gleich in einigen Gegenden Sachsens ein ziemlich bedeutender Obstbau

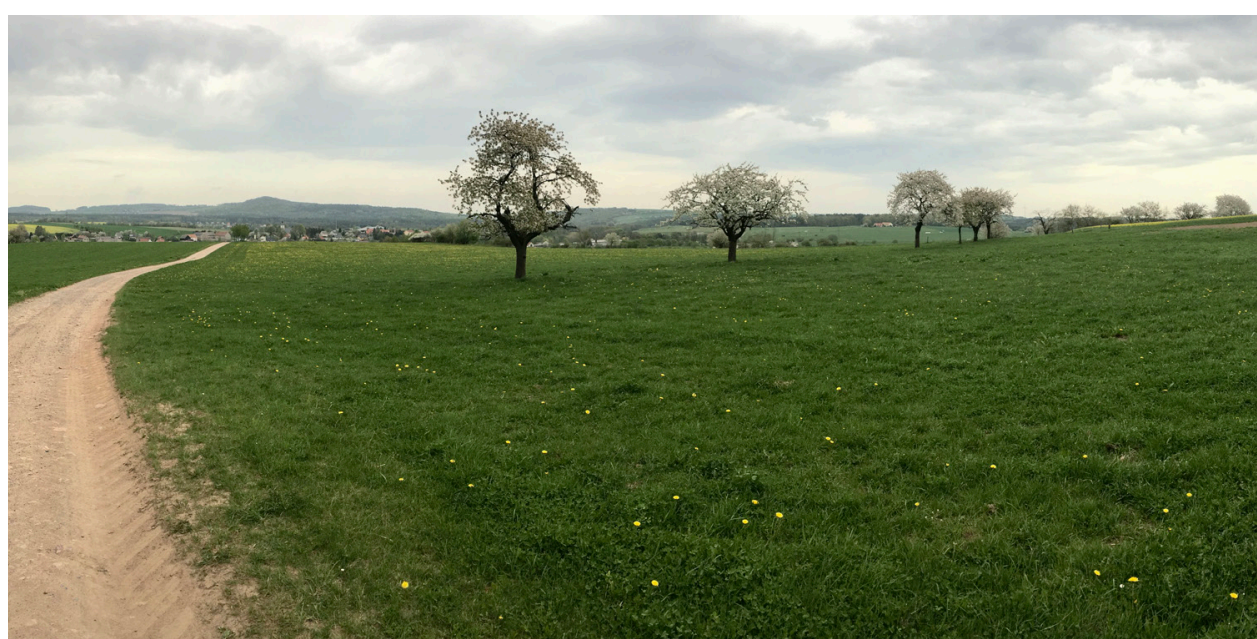

6 | Blick von Süden über die Felder mit blühenden Obstgehölzen in Richtung Reinhardtsgrimma, Fotografie: Stefan Makowski, Frühjahr 2018.

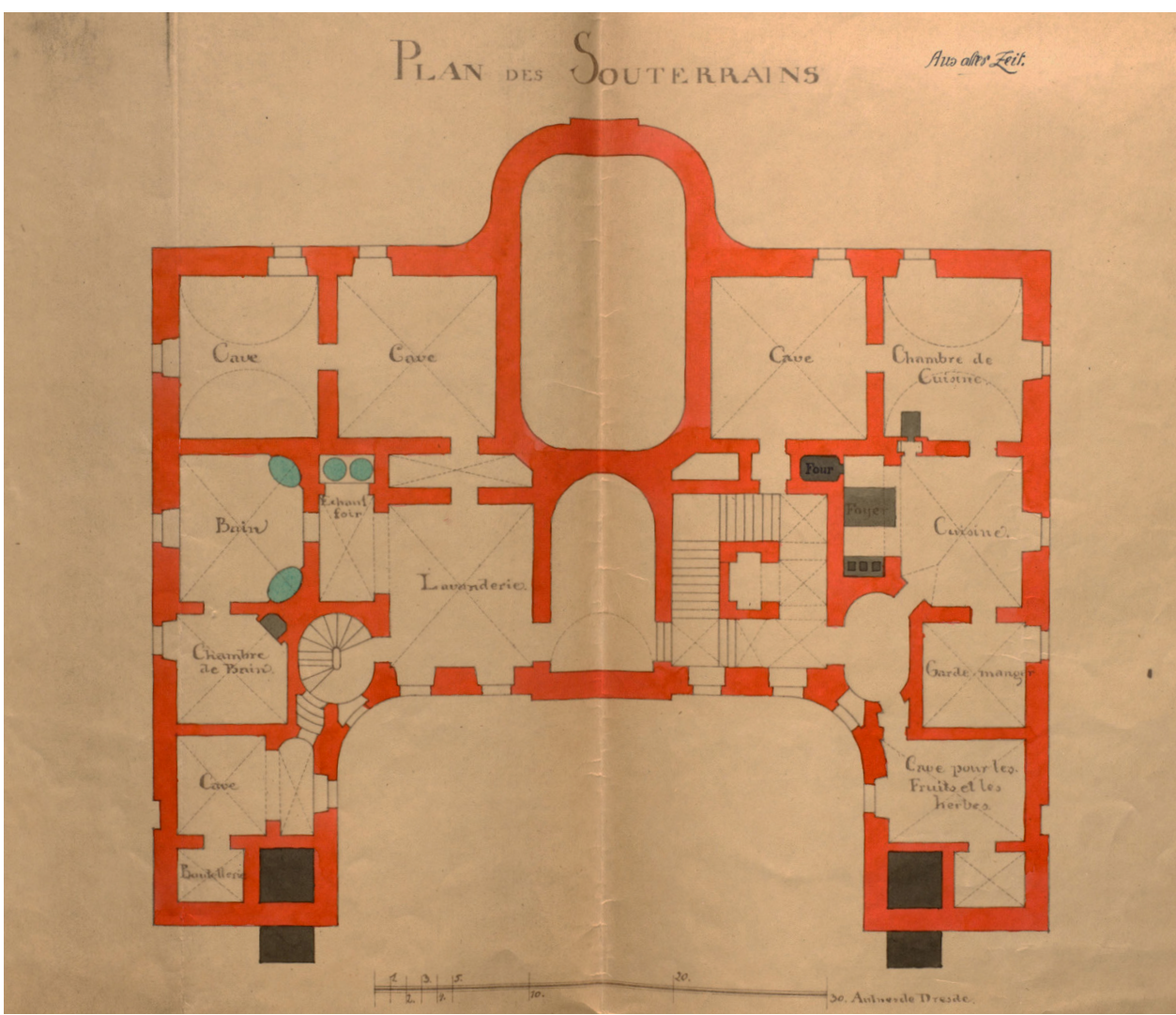

7 | Schloss Reinhardtsgrimma, "Plan des Souterrains", Johann Friedrich Knöbel, um 1767 (SächsHStA, 10505, Nr. 409, Pläne des Schlosses Reinhardtsgrimma 1907-1909).

getrieben wird, so ist doch zu bemerken, Der Obstbau nach neuesten Erkenntnisdaß derselbe bei weitem noch nicht die sen ab 1866

Bedürfnisse des Landes befriedigt und

daß der größte Theil [...] nur aus gemei- Durch Erbschaft und Heirat gelangten 1866 nen Obstsorten besteht. « ${ }^{40}$

Ludwig Emil Aster und seine Frau Friederike Conradine, geborene Ruschenbusch, in

Dies kann man wohl auch auf Reinhardts- den Besitz des Guts. Nach seinem aktiven grimma übertragen. Nichtsdestoweniger be- und erfolgreichen Militärdienst schien Aster legt der Hinweis, dass die dortige Brennerei nun seinen eigentlichen Interessen $\mathrm{zu}$ fol1842 u.a. Kirschbranntwein herstellte" ${ }^{41}$, dass gen, als er sich 1874 zum Direktor der Öko- wie man auch später immer wieder sieht - nomischen Gesellschaft im Königreich in Reinhardtsgrimma schon früh das Haupt- Sachsen wählen ließ. ${ }^{43}$ Genau in jener Zeit interesse auf der Kirschenproduktion lag. So kann man eine Neuordnung der Gutswirtzählte man 1874 insgesamt fast 2000 Kirsch- schaft in Reinhardtsgrimma ausmachen, die bäume, sowie 415 Pflaumen- und 718 Äpfel- den Idealismus der deutschen Gründerjahre und Birnbäume. ${ }^{42}$ Diese Tatsache beweist, widerspiegelnd eine Vorbildwirkung in dass der Obstbau - neben der Sicherung der Sachsen zu erlangen trachtete. Obschon Eigenversorgung - auch immer eine der Er- man Aster wegen seiner Arbeit großes Lob werbsquellen der Gutswirtschaft war. 
gewesen zu sein, um langfristig einen Zuge- Der Anbau der Gehölze in den Plantagen winn zu ermöglichen. Unter diesen Prämis- und an den Straßen erfolgte durch den Förssen muss auch die Obstkultur in Reinhardts- ter. Diesem fiel dementsprechend ein Großgrimma zu dieser Zeit betrachtet werden. teil der Arbeit zu. Obstkulturen befanden Eine wesentliche Modernisierung war das sich rund um das Gut (Abb. 8):

Flurbuch, das 1875 angelegt wurde und den

Obstbau vor Ort einbezog. Hierin zeichnete sich nicht nur eine zunehmend effiziente Wirtschaftsweise ab, sondern letztlich auch die Bedeutung, die der Pomologie zuerkannt wurde. So wird im Flurbuch und den »Beilagen zum Grundbuch und Universum für das Rittergut Reinhardtsgrimma" akribisch die Verteilung der Sorten auf den Obstplantagen verzeichnet. ${ }^{44}$ Mehr noch: Es wird ein »Obstkulturplan« entworfen, der nicht nur eine Kartierung und Markierung der Bäume beinhaltet, sondern auch ein Regelwerk enthält, wie man >Anbau und Behandlung von Obstplantagen $`$ zu bewerkstelligen habe. Diese informative Instruktion, die heute noch im Gutsarchiv vorhanden ist, mag als ein Beleg für den hohen Anspruch Asters gelten. Zum ersten Mal erhalten wir auch Auskunft über den konkreten Obstbestand. Dies ist umso interessanter, da in Bleistift ausgeführte Randnotizen in den Akten belegen, dass man aus einer handschriftlichen Vorschlagsliste geeignete Sorten herausarbeitete. Wer diesen ursprünglichen Plan erstellte, wird allerdings nicht deutlich. Doch darf man davon ausgehen, dass die wesentlichen Ideen zur Neuausrichtung der Obstkultur in Reinhardtsgrimma direkt aus dem Umfeld der Ökonomischen Gesellschaft stammten.

\section{Das Flurbuch und der Obstbau}

Ein Obstkulturplan schaffte Überblick übe die Anpflanzungen und deren Anordnung. Der Anbau des wertvollen Tafelobstes, wie lag nicht dem Gärtner, sondern vielmehr beispielsweise Pfirsiche und Aprikosen, aber dem Förster. Auch wurden der Anbau und auch Tafelwein, Beerenobst und derglei- die Behandlung von Obstplantagen klar dechen, wurde durch den Schlossgärtner be- finiert. In den Akten werden Arbeiten wie stellt, der durch zahlreiche Spaliere nicht das Schachten von Löchern, das Zurücknur den Hortosol, sondern auch die Sonne optimal zu nutzen wusste. ${ }^{45}$ an der Frauendorfer Straße, am Hang bei den Bergschluchten vom Oberhof bis Dorfgrund sowie am Hang des Quergrundes am Ernestinenfelde vom Oberhof bis hin zum Quergrund

am Oberhofgarten links- und rechtsseitig, als auch rund um die Drescherhäuser bis hin zum Grund, entlang der Buschhausstraße bis zur Waldgrenze sowie der Folge am Liebstein

entlang der Schusterbrücke längs der Feldwiese, ebenso wie bei den Dreispitzen bei der Hirschbachgrenze und der Schafmeisterei sowie der Hirschbachallee

innerhalb und außerhalb des Mauergartens sowie an der Försterei, der Schäferei, dem Pflanzgarten und auch im Wäschegarten. ${ }^{46}$

Aus dem Flurbuch ist zu entnehmen, dass an der Straße Richtung Frauendorf Kirschbäume standen. ${ }^{47}$ Auch zum gegenwärtigen Zeitpunkt begleiten alte Kirschbäume Bereiche der Straße Richtung Frauendorf. Jedoch kann nicht mit Bestimmtheit festgestell werden, ob diese tatsächlich einem Alter vo Jahre entsprechen und demnach aus der Zeit des regen Obstanbaus um Reineres Weiteren sind an der Straße Richtung Luchau augenscheinlich Kirschen vorhanden, diese wurden jedoch im Flurbuch von 1875 nicht erwähnt.

schneiden von Wasserreißern, das Aufasten, das Weißen der Bäume aufgezählt, wobei das Pfropfen als Veredlungsmaßnahme und

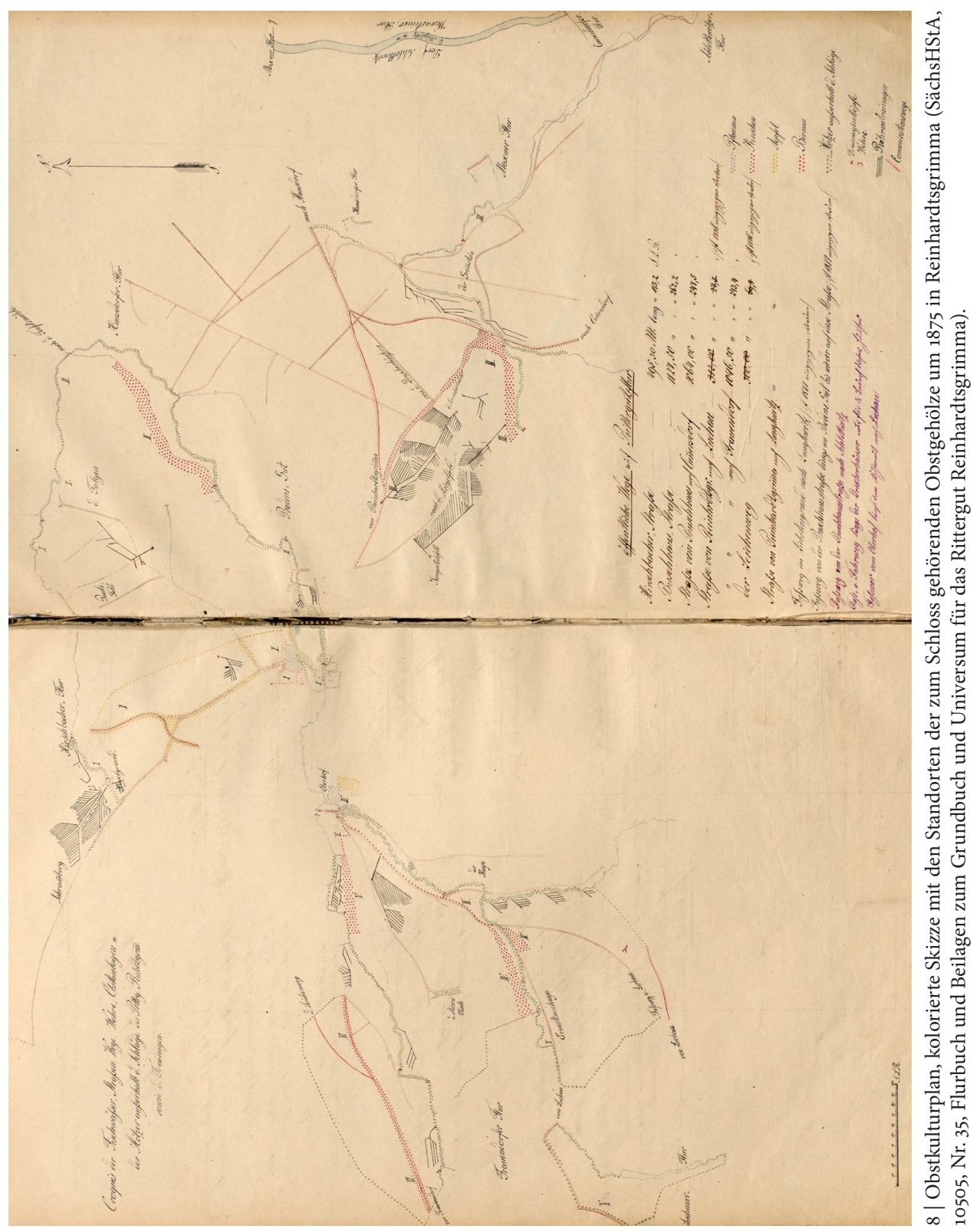


meistens auch Hilfskräfte hinzugezogen wurden, welche angewiesen waren die Gehölze zu schützen, zu pflegen und zu nummerieren. Des Weiteren sollte ein Verzeichnis derjenigen Obstsorten angelegt werden, welche ab dem Jahr 1875 ausschließlich auf dem Rittergut Reinhardtsgrimma angebaut werden sollten (Abb. 9)..$^{48}$

Interessant ist auch, dass sich laut dem 1874 angelegten Register - über das bewegliche Inventar des Rittergutes Reinhardtsgrimma hinaus - im Forsthaus neben den aufgeführten Hilfsmitteln zum Obstbau wie Obststeckpfählen und Obstleitern auch eine Obstveredelungskarte sowie ein Obstkulturplan mit Karte befanden. Zudem wird im Inventar auch Literatur des schon genannten Pomologen Eduard Lucas erwähnt. ${ }^{49}$

Aus dem »Verzeichnis der Obstsorten welche vom Jahr 1875 ausschließlich auf dem Rittergute Reinhardtsgrimma angebaut werden sollen $\aleph^{50}$ wissen wir recht genau über die Sortenzusammenstellung vor Ort Bescheid. Es werden verschiedene Apfelsorten aufgeführt und auch Synonyme angegeben, ebenso wann sie >zeitigen`, d.h. ob es sich um Winter-, Frühjahr-, Herbst- oder Sommeräpfel handelt. Darüber hinaus wurde ihre Funktion als Tafel- oder Wirtschaftsapfel angegeben. Teilweise ging man auch auf Wuchsbedingungen, Haltbarkeit und Bodenansprüche ein. Auffällig ist, dass Äpfel das ganze Jahr zur Verfügung standen, da durch die geschickte Sortenwahl eine Lagerfähigkeit bis in den nachfolgenden August gewährleistet war, wenn die Klaräpfel eine neue Saison einleiteten. ${ }^{51}$ Genannt werden 20 Sorten $^{52}$, darunter:

»Rother Eiserapfel, Großer rheinischer (gelb- eine Übersicht über die Einnahmen und grün), Große Casseler Reinette (gelb), Ausgaben durch den Obstbau, z. B. aus dem englische Scharlachrothe Parmäne, Engli- wöchentlichen Verkauf. Dabei wird deutsche Wintergold-Parmäne, Golden Sta- lich, dass mit dem Verkauf von Zierpflanzen ben (?), Weißer Astrachaner, Rother Ast- zunächst mehr Einnahmen erzielt wurden rachen, Langtons Sondergleichen, gelbe als mit dem Verkauf bzw. aus dem Ertrag gestreifte Schafsnase, kleiner Herrenapfel, von Nutzpflanzen. Der Ankauf beim Obst-

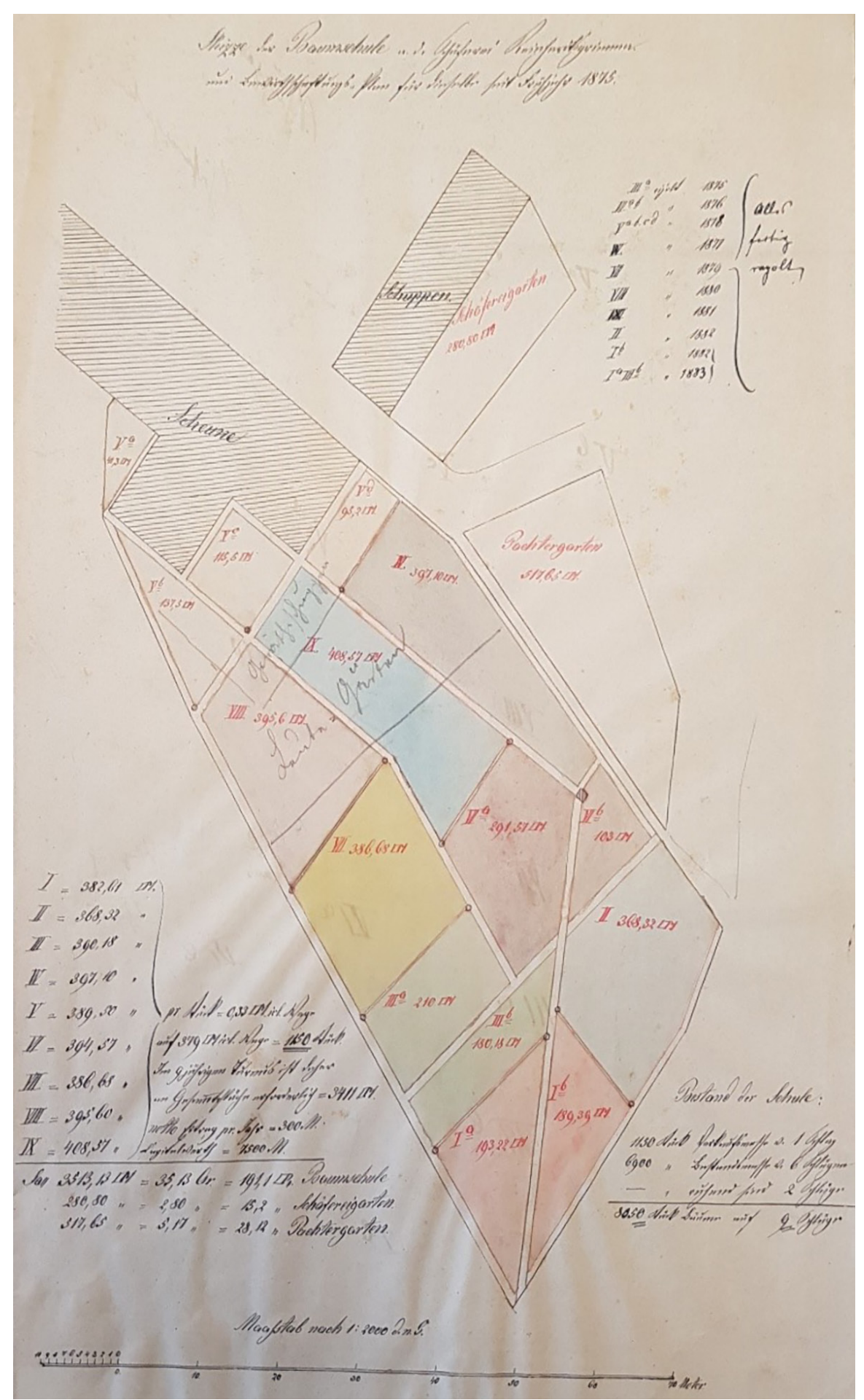

9 | Skizze der Baumschule und Gärtnerei am Schloss in Reinhardtsgrimma um 1875, kolorierte Skizze, (SächsHStA, 10505, Nr. 400 Wirtschaftsplan und Skizze der Baumschule und Schäferei von Reinhardtsgrimma, 1887) 
bau bezog sich hauptsächlich auf Edelobst- ort und jedes Spalier und betont die 3.000 reiser, so konnte man beim Gutsförster ein-bis fünfjährigen Obstbäume in der Hanke beispielsweise Kirschedelreiser käuf- Baumschule. ${ }^{59}$ Jahre später zählte man beim lich erwerben..$^{5}$ Verkauf dort bereits 8.050 kleine Bäume. ${ }^{60}$

\section{4 erfolgte eine Obstbaumzählung der Die Obstwirtschaft nach den Asters} weiter oben aufgeführten Standorte. So

zählte man auf allen zum Gut gehörenden Seit der Gründerzeit wurde der Obstanbau Ländereien

"außerhalb des Gebäudes:

- 63 Kirschbäume I a. b.

- 316 dgl. I c-i

- 143 dgl. I k

- 560 dgl. II a-i

- 70 Pflaumenbäume II a.b

- 85 Birnenbäume III

- 93 Pflaumenbäume und Quitten in Ober.Schloßgarten links III b

- 87 Pflaumenbäume incl. 19 Spalierbir nen in Ober. Schloßgarten rechts III c

- 84 Apfel-, Birn-, Kirschbäume im Mauergarten excl. des Weges III d

- 440 Apfelbäume an dem Dippoldiswalde und Hirschbachweg incl. [...] III $\mathrm{e}-\mathrm{n}$

- 90 Apfelbäume und Birnen im Oberhofgarten links IV a

- 185 Pflaumenbäume im Oberhofgarten rechts IV b

- 154 Kirschbäume am Luchwege und Quergrund IV c

- 168 Kirschbäume an der FrauendorferStraße V a-c ${ }^{56}$

Dazu kamen diverse Obstspaliere am Gärtnerwohnhaus, dem Bienenhaus, dem Kalthaus und dem Forsthaus. ${ }^{57}$ Die Akten nennen zudem den Ankauf von 39 Obstbäumen, die Pflanzung von 443 Obstbäumen und das Beharken von 1.807 Obstbaum scheiben, was die Dimension und Wichtigkeit des Obstbaus auf Reinhardtsgrimma noch einmal unterstreicht. ${ }^{58}$ als wichtiger Wirtschaftsfaktor gesehen und intensiviert. Erstmals bepflanzte man im großen Umfang Felder und Wiesen systematisch. Man übertrug das vorangegangene Anbausystem der Wege- und Saumbepflanzung in die Landschaft. Vorherrschend blieb bis zum Zweiten Weltkrieg der Hochstammobstbau. ${ }^{61}$ Es kam zur Rationalisierung und Produktionssteigerung. Man kultivierte erfolgreich Zwergobstkulturen, allerdings fanden diese hauptsächlich in den Produktionsstätten des Intensivobstbaus Verwendung. Maßgebend war eine leichte Erreich barkeit. So eingehend man sich in Reinhardsgrimma auch mit dem Obstbau beschäftigte, so war dies dennoch nicht der einzige Wirtschaftszweig und ist mit den Intensivobstbaustätten zu jener Zeit nicht vergleichbar.

Auch unter dem neuen Besitzer, dem Ökonomierat Johann Maximilian Nitzsche wurde die Obstwirtschaft weitergeführt. Das "Capitel außerhalb des Forstes Reinhardtsgrimma « ${ }^{62}$ von 1885 bis 1887 gibt Auskunft über die Einnahmen aus der Verpachtung der Obstbäume und dem Verkauf von Obst. So befindet sich darin ein tabellarisches Verzeichnis über Einnahmen und Ausgaben der Obstwirtschaft. Einnahmen generierte man über den Verkauf von Obst, den daraus gewonnenen Erzeugnissen als auch aus de Obstpacht. Ausgaben wurden getätigt für Material, Lohn und Arbeiten in den Plantagen rund um das Rittergut (vgl. Beitrag »Die Entwicklung der Gärten «). ${ }^{63}$ Hinzu kam, Obschon ein direkter Vergleich schwierig dass Rittergutsbesitzer Nitzsche 1891 den köist, so wird dennoch eine Intensivierung der niglich-sächsischen Gartenbaudirektor und Obstbaumzucht unter den Asters aus den Gartenkünstler Max Bertram beauftragte eiAkten ablesbar: Das überaus differenzierte nen Änderungsvorschlag für den SchlossInventar von 1874 verzeichnet jeden Stand- garten zu unterbreiten. ${ }^{64}$ Letzterer galt als ein Förderer der Pomologie, dessen Credo lautete: »Gärten sind kein Luxus, sondern Erfordernis $\ll^{65}$

Vor dem Verkauf Reinhardtsgrimmas durch die Witwe Nitzsches an Friedrich Hugo Maximilian Oberst Senfft von Pilsach und dessen Frau wurde in einem Gutachten vom 6 Juli 1907 die Gesamtfläche des Parks und aller Gärten mit zehn Hektar angegeben. ${ }^{66}$ Des Weiteren erfasste das Gutachten die Obstpacht, die jährlich 330 Reichsmark einbrachte, sowie die Einnahmen der Gutsgärtnerei, die laut Schriftstück jährlich 1.000 Reichsmark abwarf.

Auch unter von Senfft wurde akribisch Buch über Einnahmen und Ausgaben geführt. So bestätigen die Gärtnereikontobücher von 1908 bis 1912 zwar Einnahmen durch Obst, Obstbäume und Honig, jedoch mussten Obstpflücker engagiert werden, um bei der Ernte mitzuhelfen. Ersichtlich ist aus dieser Dokumentation ebenfalls, dass man die Obstbäume nicht nur selbst bewirtschaftete sondern auch verpachtete. Darüber hinaus scheint man junge Obstbäume aus der Baumschule verkauft zu haben. In Reinhardtsgrimma kam es 1921 im Übrigen zu einem Rechtsstreit der Senfft von Pilsachs mit Einheimischen, da Leute aus dem Dorf die Obstbäume an den Wegen des Rittergutes abernteten, nachdem sie aufgrund der Kommunalisierung des Straßenbaus der irrigen Annahme waren dieses Obst sei Allgemeingut. ${ }^{67}$ Weiterhin geben die Akten Auskunft, dass sich eine kleine Zahl an Bienenstöcken für den Obstbau in der Obhut des damaligen Schlossgärtners Georg Karliczek befand. ${ }^{68}$ Die Inventarliste vom 31. März 1939 führt »6 Bienenbauten, hölzern (2 mit Bienen) « ${ }^{69}$ auf. Hinzu kam die Grundausstattung wie Honigschleuder, Bienenhaube, Wabentablett, eine Bienenpfeife, ein Honigmesser und eine Wabenzange. Die Imkere fiel also in das Tätigkeitsfeld des Gärtners.

Daneben musste man den Bestand aber auch durch Zukäufe erhalten: so erwarb man 1909 beispielsweise 500 Apfelwildreiser und 500 Kirschbäume, deren Anzahl weit über den Eigenbedarf hinausging..$^{70}$ Bezogen wurden die größeren Gehölze u.a. aus dem böhmischen Tetschen, dem fränkischen Hofstetten sowie der Baumschule Erns Pflanz in Böhlen bei Leipzig, die auf hochstämmige Obstgehölze, Formbäume, Beerenobst und Ziergehölze spezialisiert war aber auch Entwürfe ausführte..$^{71}$ Von hier bezog Oberst Senfft von Pilsach für das Schloss Reinhardtsgrimma unter anderem zwölf Apfel-Pyramiden (Abb. 10)..$^{72}$

Aus den vorhandenen Personalakten der Gärtner wird deutlich, dass Kenntnisse der Obstbaukunde unerlässlich waren: Der Gärtner Karliczek lobte in seinem Bewerbungsschreiben seine Kenntnisse der Weinund Pfirsichtreiberei; der Gärtner Hinz erwähnte ausdrücklich, dass er in Süddeutschland den Formobstschnitt nach Johann Ludwig Christ und Eduard Lucas erlernt hätte (vgl. Beitrag »Die Gutsgärtner von Reinhardtsgrimma

Zwischen 1928 und 1933 lösten vermutlich klimatische Bedingungen einige Missernten vor Ort aus, zudem wirkten sich Teuerungen und die Wirtschaftskrise negativ auf die Gutswirtschaft aus. So ist neben Lohnteuerung und Entlassungen auch von einer geringen Obsternte die Rede, von Spitzendürre und einer großen Trockenheit, die das Obs von den Bäumen fallen ließ. Das Obst an den Alleen fiel dürftig aus und es musste hart bei der Schädlingsbekämpfung har vorgegangen werden. Andererseits standen Obstbäume und Beerensträucher im Frühahr in voller Blüte, so dass Einnahmen aus dem Verkauf von Frühobst verzeichnet wurden und man für den Eigengebrauch einweckte. Man investierte weiterhin in die Neuanpflanzung von Obstbäumen. So kam es 1930 zur Setzung von 100 Obstbäumen in den Garten sowie von 133 Alleebaumen entlang der Straßen, hauptsächlich Äpfel und Kirschen. ${ }^{73}$ 


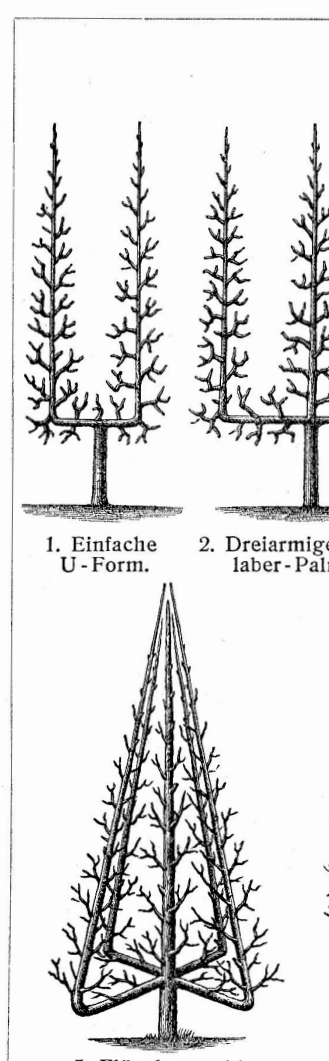

5. Flügelpyramide.

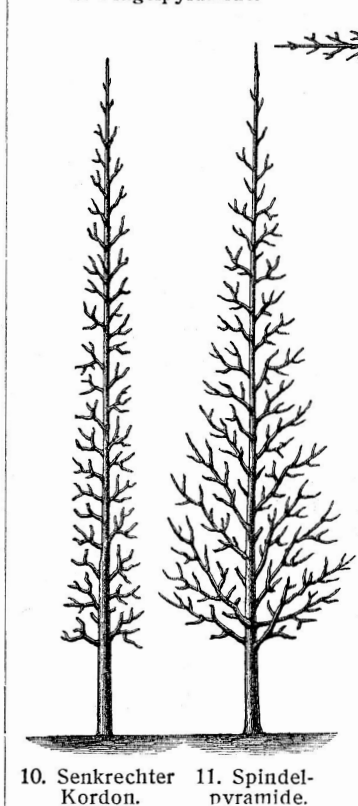

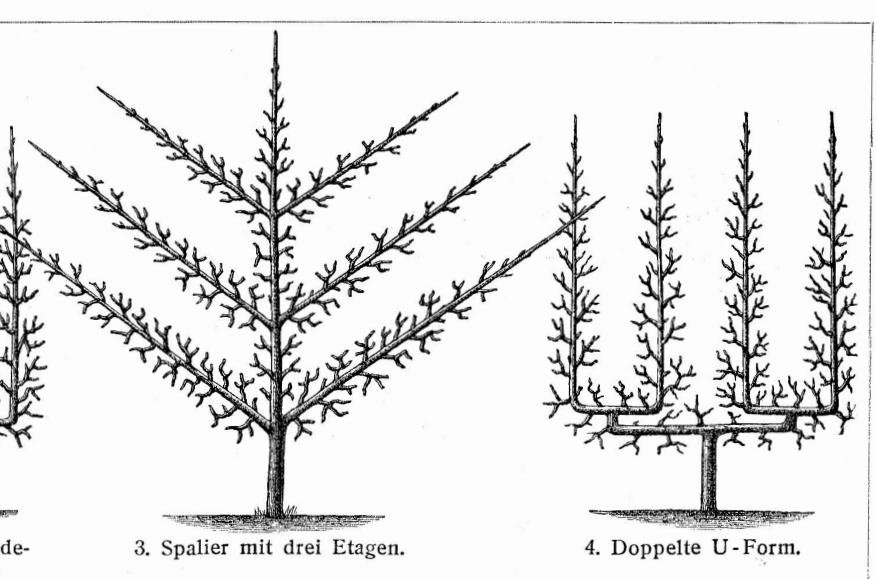

Doppelte U-Form.

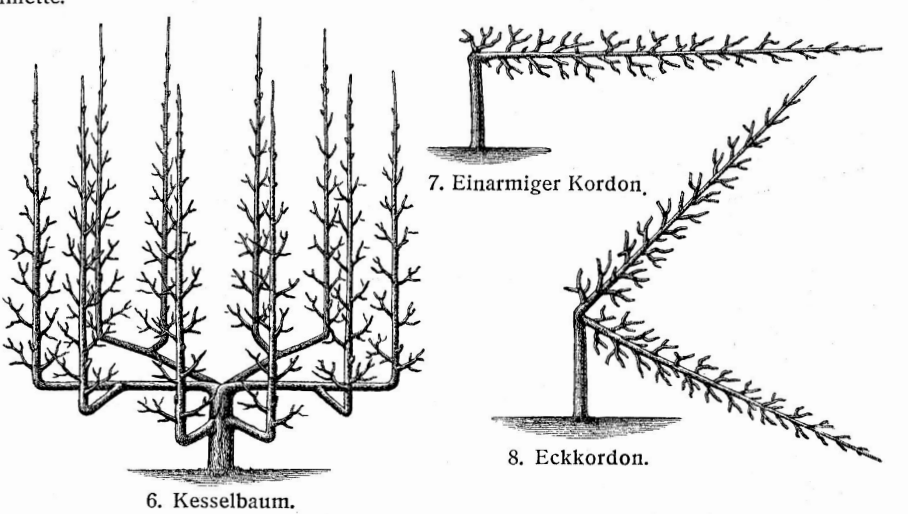

6. Kesselbaum.

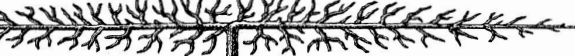

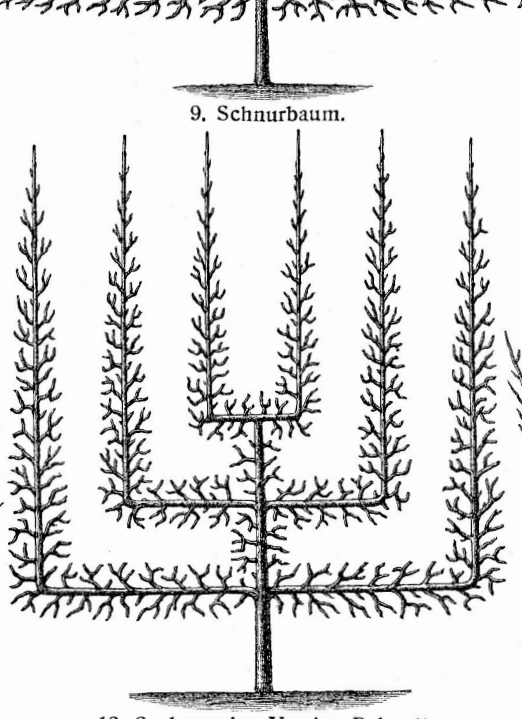

12. Sechsarmige Verrier - Palmette.

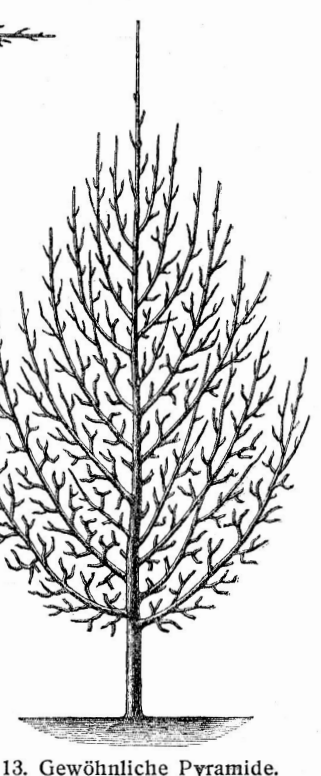

13. Gewöhnliche Pyramide.

o | Darstellung kunstvoll erzogener Obstbäume, welche durch gezielte Schnitt- und Bindemaßnahmen in ihre Wuchsform geleitet worden sind. Abbildung Zwergobstbäume, (Meyers Großes Konversations-Lexikon, Band 14 Leipzig 1908, S. 883).

Auch nach dem Tod Maximilian Senfft von spielsweise Freiherr von Friesen in Rötha Pilsachs1931 wurde die Obstwirtschaft bis entwickelte, der nicht nur die wachsende zur Enteignung 1945 fortgeführt. So waren Großstadt Leipzig mit frischem Obst und die Rittergutsbesitzer 1939 vom Bürgermeis- Obstsaft versorgte, sondern seinen Landster der Gemeinde angehalten circa 200 leuten auch durch Publikationen VerbesseObstbäume in Ordnung zu bringen, nach- rungen vorschlug, muss auch auf Rein dem dieser bei einer Besichtigung der Obst- hardtsgrimma ausgestrahlt haben.

gärten und Anlagen Handlungsbedarf fest- Dass man Anschluss an das aktuelle Zeitgestellt hatte. Man kennzeichnete die Bäume, geschehen suchte, mag die überkommene um auf die entsprechenden Maßnahmen Druckschrift »Die Bezirks-Obstbau-Aushinzuweisen. Dieses Schreiben gewährt er- stellung in Dippoldiswalde am 8. und 9. Ocneut einen Einblick in die Weitläufigkeit der tober 1876 « belegen, da dort einige Apfelzum Rittergut gehörenden Obstkulturen. ${ }^{74}$ und Birnensorten angestrichen sind und So befanden sich laut Inventarverzeichnis sich zudem Karl Alexander von Langsdorffs der Gärtnerei Reinhardtsgrimma am 31. Schrift »Obstsorten für rauhere Gegenden « März 1939 auf deren Flächen 468 Apfel, 16 in der Akte findet. ${ }^{77}$ In dessen Aufsatz wird Birnen, 155 Kirschen, 16 Sauerkirschen, 63 auch deutlich, dass die Pomologie damals Pflaumen sowie 506 Stachel- und Johannis- fast volkstümlichen Charakter hatte. So finbeeren. ${ }^{75}$ Obstgehölze auf den Ländereien den sich unter den 2.500 Mitgliedern des rings um das Schloss nutzten die Gutsbesit- 1874 gegründeten Landesobstbauvereins zer. Zusätzliche Einnahmen wurden sowohl Sachsen als Förderer bekannte Persönlichdurch die Verpachtung von Obstgehölzen keiten, wie der

an die Gemeinden Reinhardtsgrimma, Hirschbach und Hausdorf als auch durch Alleebäume an den Dorfstraßen wie beispielsweise der Frauendorfer Straße erzielt. Aber auch die Verpachtung von Obstgehölzen an Einzelpersonen in Tharandt, Klingenberg, Dittmansdorf oder Niedersedlitz war üblich und brachte weitere Einnahmen zu Gunsten des Gutes mit sich. ${ }^{76}$

\section{Fazit}

Anhand der Entwicklung der Obstkultur in Reinhardtsgrimma lässt sich die Geschichte In diese Reihe von interessierten Laien und der sächsischen Pomologie nachvollziehen. Spezialisten lassen sich auch die Besitzer Wenngleich auch zuweilen Mitläufer, so von Reinhardtsgrimma einordnen. Man zeichnet sich gegen Ende des 19. Jahrhun- spielte dabei keine herausragende Rolle, war derts das Bestreben ab, das Obst als eine si- jedoch stets auf der Höhe der Zeit, was sich chere Einnahmequelle in die Gutswirtschaft auch heute noch anhand des Gutsarchivs einzubeziehen. Die Vorbildwirkung, die bei- nachvollziehen lässt. 
1 SächsHStA, 10505, Nr. 521, Kauf des Rittergutes Reinhardtsgrimma durch Johanna Joachime Charlotte Racknitz, 1800

2 Döge, Regina: Die Wurzel des sächsischen Obstbaus, Ein historischer Abriss zur Entwicklung des Obstbaus vom Mittelalter bis zur Gegenwart, PirnaBirkwitz 2010, S. 1.

3 Wimmer, Clemens Alexander: Geschichte und Verwendung alter Obstsorten, Deutsche Gesellschaft für Gartenkunst und Landschaftskultur (DGGL) e.V., Berlin 2003, S. 22.

4 Weitergehende Informationen dazu finden sich im Absschnitt >Der Obstbau nach neuesten Erkenntnissen ab 1866.

5 Balling, Edwin: Die Kulturgeschichte des Obstbaus, in: https://www.lfl.bayern.de/mam/cmsoz/iab/ dateien/ kulturgeschichte_obstbau_extern.pd (Zugriff am: 20.02.2020)

6 Wimmer 2003, S. 22ff.

7 Sammlung von Gesetzen in Kursachsen, in: Lingelbach, Gerhard: Der »Codex Augusteus« - zu Entstehung, Inhalt und Wirkungsgeschichte einer (fast) vergessenen Rechtssammlung, in: Lück, Heiner Schildt, Bernd (Hg.): Recht, Idee, Geschichte: Beiträge zur Rechts- und Ideengeschichte für Rolf Lieberwirth anläßlich seines 80 . Geburtstages, Köln 2000, S. 249-274

8 Döge 2010, S. 1.

Nagel, Christine: Obst- und Gartenbau unter Kurfürst August von Sachsen (1526-1586), in: Aha! Miszellen für Gartengeschichte und Gartendenkmalpflege, Heft 5, Dresden 2019, S. 8-17, hier S. 10.

10 Kober-Carrière, Ragnhild; Wimmer, Clemens Alexander: Ein Dresdner Obstsortenverzeichnis von 1594, in: Zandera. Magazin der Bücherei des Deutschen Gartenbau Vereins e.V.,Band 25 (2010), Nr. 2, S. 94.

11 Churfürst August zu Sachsen: Künstlich Obstgarten-Büchlein, nach der letzten Original-Ausgabe v. J. 1620 für Liebhaber der Pomologischen Literatur aufs neue, mit Anmerkungen des Herrn Hof- und Kanzl.-Rath v. Laffert zu Celle, Weimar 1802.

2 Döge 2010, S. 2

13 Wimmer 2003, S. 28

14 Döge 2010, S. 3.

15 Ebd.

6 Verzeichnis der Obstsorten in der systematischen Obstbaumschule im königl. Sächs. großen Garten bei Dresden, Dresden 1819. Es ist auffällig, dass nur zwei Prozent der Schriften der Ökonomischen Sozietät in Leipzig zwischen 1764 und 1814 dem Obstbau gewidmet sind. Erst in der Zeit nach den Befreiungskriegen nahm das Interesse am Thema merklich zu, vgl:: Die Leipziger ökonomische Sozietä 1763 bis 1825: Horst-Springer-Stiftung für Neuer Geschichte Sachsens in der Friedrich-Ebert-Stiftung; Rede anlässlich der Verleihung des HorstSpringer-Preises 1998 / Andreas Schöne - [Electronic ed.] - Bonn, 2001 - http://library.fes.de/fulltext/ historiker/01081.htm (Zugriff am: 08.07.2020).

17 Vgl. Meilenblätter von Sachsen, Dresdner Exemp- lar, Blatt 309: Dresden, 1781 (Deutsche Fotothek, Signatur dd_hstad-mf_oooo328).

18 Döge 2010, S. 4.

Landschaftspflegeverband Nordwestsachsen e.V. Vergessene Vielfalt-Streuobstwiesen in Sachsen, in https://www.streuobst-in-sachsen.de (Zugriff am: 19.10.2020).

20 Wimmer 2003, S. 18.

Historische Zeitschrift des Deutschen Pomologenvereins, 1855-1905, gemeinsam herausgegeben mit Johann Georg Conrad Oberdieck (evangelischer Pfarrer und Pomologe), in: Fehleisen, Phillip: Biographische Skizze mit Porträt, in: Oberdieck, Johann Georg Conrad; Lucas, Eduard: Illustriert Monatshefte für Obst- und Weinbau - Organ des Deutschen Pomologen-Vereins, Ravensburg 1867, S. 129-134.

22 Döge 2010, S. 4.

23 Wimmer, Clemens Alexander: Die Freiherr von friesensche Sammlung - Ein Denkmal der Pomologie in der Gartenbücherei, in: Zandera 18, Nr. 1 2003, S. 3-4. Siehe auch: Gottschalk, Anja: Das gartenkulturelle Erbe von Rötha. Neueste Erkenntnisse zur Entwicklung des Schlossgartens im 17. und 18. Jahrhundert, in: Landesamt für Denkmalpfleg Sachsen (Hg.): Mitteilungen des Landesamtes für Denkmalpflege, Jahrbuch 2019, S. 16-27.

24 Döge 2010, S. 9.

Empfehlungen des Bezirks-Obstbau-Vereins zu Dresden als zum allgemeinen Anbau an öffentlichen Straßen und Chausseen in Sachsen geeigneten Apfel- und Birnensorten, in: Deutsche Garten- und Obstbauzeitung, 1879, S. 74-75.

26 Döge 2010, S. 9.

28 Balling, Edwin: Die Kulturgeschichte des Obstbaus, n: https://www.lfl.bayern.de/mam/cmsoz/iab/ dateien/kulturgeschichte_obstbau_extern.pdf (Zugriff am: 20.02.2020)

29 Willig, Hans-Peter: Obstnutzung, in: https://www. biologie-seite.de/Biologie/Obstnutzung (Zugriff am: 19.10.2020).

SächsHStA, 10505, Nr. 541, Erbregister, 1624

31 Ebd.

32 Ebd., Nr. 400, Skizze und Wirtschaftsplan der Baumschule und Schäferei von Reinhardtsgrimma, 1875 .

Ebd., Nr. 135, Pachtvertrag zwischen Eleonore Christiana von Tettau, geb. von Berbisdorf, au Reinhardtsgrimma und Georg Abraham Siegert über das Rittergut Reinhardtsgrimma, 1716-1731.

4 Ebd., Nr. 179, Gärtnerbestallung Johann Friedrich Reuter, 1809.

35 Ebd.

Ebd., Nr. 409, Pläne des Schlosses Reinhardts grimma, 1907

38 Ebd., Nr. 250.

40 Flotow, Gustav von: Uber die Beforderung des

Obstbaues, besonders über die Verbreitung edler
Obstsorten in Sachsen, in: Schriften und Verhand- 57 Ebd. lungen der ökonomischen Gesellschaft im Kön reiche Sachsen,Band 24, Dresden 1830, S. 79.

41 SächsHStA, 10505, Nr. 309, Wirtschaftsrechnungen,

42 Ebd., Nr. 34, A unbewegliches Inventar bei dem Rittergute Reinhardtsgrimma, 1874.

43 Ende, Ernst am: Die Oekonomische Gesellschaft in Königreich Sachsen in ihrer geschichtlichen Entwicklung seit 120 Jahren, Dresden 1884, S. 3. Dieses Buch ist »Herrn Ludwig Emil Aster Hauptmann zu Dresden, dem verdienstreichen Direktor der Oekonomischen Gesellschaft im Königreiche Sachsen während der letzten 10 Jahre als Ausdruck hohe Achtung gewidmet«.

44 SächsHStA, 10505, Nr. 35, Flurbuch und Beilagen Reinhardtsgrimma 1875 .

45 Ebd., Nr. 77, Einnahmen und Ausgaben, 1875-1877. 46 Ebd., Nr. 184, Verzeichnis der Obstsorten vom Jahr 1875,1875 .

47 Ebd., Nr. 35

48 Ebd.

9 Ebd., Nr. 26, bewegliches Inventar 1874, 1874. Do ist "Lucas Obstbau « verzeichnet, gemeint ist möglicherweise Eduard Lucas populäre »Anleitung zu Obstkultur « (1869)

50 Ebd., Nr. 184

1 Zu den Haltbarkeiten, siehe: Brudel, Fritz; Kaless Andreas: Obstatlas der Russischen Kolonie Alexandrowka in Potsdam - ein pomologischer Führe Potsdam 2012, S. 53-300.

52 SächsHStA, 10505, Nr. 18

53 Schlitt 2019, S. 32-35.

ür rauhere Gegenden, in: Deutsche Garten- und Obstbauzeitung, 1879, S. 91-93.

55 SächsHStA, 10505, Nr. 77

56 Ebd., Nr. 34.
Ebd., Nr. 66, Capitel außerhalb des Forstes Rein hardtsgrimma, 1882-1885

59 Ebd., Nr. 35.

60 Ebd., Nr. 400

61 Wimmer 2003, S. 35.

2 SachsHStA, 10505, Nr. 67, Capitel außerhalb des Ebd.

64 Entwurfsplan von Bertram für den Park in Rein hardtsgrimma: LfDS, Plansammlung, Inv.-Nr. 2018-

65 Lux, Andreas Rene: Gärten sind kein Luxus, sondern Erfordernis: zum 150. Geburtstag des Gartenkünstlers Max Bertram, in: Dresdner Neueste Nachrichten: DNN; die Union, Verlag Dresdne S. 14 .

66 SächsHStA, 10505, Nr. 104, Baurechnungen, 19071909 .

67 Ebd., Nr. 477, Rechtsstreit über Nutzung von Obstbäumen entlang der Dorfstraße, 1921.

b., Nr. 329, Anstellung und Tatigkeit des Gärtner Joseph Hinz in Reinhardtsgrimma 1927-1928.

Ebd., Nr. 264, Forst- und Gärtnerangelegenheiten von Reinhardtsgrimma, 1933-1939.

70 Ebd., Nr. 27, Gärtnereikontobuch 1908-1912.

71 Ebd., Nr. 121, Gärtnerei- und Parkrechnungen, 1909-1910.

73 Ebd., Nr. 127, Gärtner Reinhardtsgrimma, 1928-1933. 74 Ebd., Nr. 264, Forst- und Gärtnereiangelegenheiten, 1911-1940.

75 Ebd.

76 Ebd., Nr. 27, Gärtnereikontobuch 1908-1912. Ebd., Nr. 184, Verzeichnis der Obstsorten vom Jah 1875, 1875; erstmals erschienen in: Sächsische Landwirtschaftliche Zeitung, 1879, S. 91ff. 